Research Article

\title{
Type 2 Diabetic Sepsis Patients Have a Lower Mortality Rate in Pioglitazone Use: A Nationwide 15-Year Propensity Score Matching Observational Study in Taiwan
}

\author{
Ming-Shun Hsieh $\mathbb{D}^{1,2,3,4}$ Sung-Yuan Hu, ${ }^{3,4}$ Shu-Hui Liao, ${ }^{5}$ Chia-Ming Chang $\mathbb{D},{ }^{2,3}$ \\ Vivian Chia-Rong Hsieh $\mathbb{D}^{6},{ }^{6}$ and Chorng-Kuang How $\mathbb{D}^{2,3,7}$ \\ ${ }^{1}$ Department of Emergency Medicine, Taipei Veterans General Hospital, Taoyuan Branch, Taoyuan, Taiwan \\ ${ }^{2}$ Department of Emergency Medicine, Taipei Veterans General Hospital, Taipei, Taiwan \\ ${ }^{3}$ School of Medicine, National Yang Ming Chiao Tung University, Taipei, Taiwan \\ ${ }^{4}$ Department of Emergency Medicine, Taichung Veterans General Hospital, Taichung, Taiwan \\ ${ }^{5}$ Department of Pathology and Laboratory, Taipei Veterans General Hospital, Taoyuan Branch, Taoyuan, Taiwan \\ ${ }^{6}$ Department of Health Services Administration, China Medical University, Taichung, Taiwan \\ ${ }^{7}$ Kinmen Hospital, Ministry of Health and Welfare, Kinmen, Taiwan
}

Correspondence should be addressed to Vivian Chia-Rong Hsieh; hsiehchiarong@gmail.com and Chorng-Kuang How; ckhow@ vghtpe.gov.tw

Received 15 May 2021; Accepted 6 July 2021; Published 23 July 2021

Academic Editor: Yan-Ren Lin

Copyright ( $) 2021$ Ming-Shun Hsieh et al. This is an open access article distributed under the Creative Commons Attribution License, which permits unrestricted use, distribution, and reproduction in any medium, provided the original work is properly cited.

\begin{abstract}
Background. Pioglitazone use via the PPAR $\gamma$ agonist in sepsis patients is inconclusive. It was based on a great number of animal studies. However, except for information from animal studies, there are merely any data of human studies for reference. Methods. This study was conducted by a unique database including 1.6 million diabetic patients. From 1999 to 2013, a total of 145,327 type 2 diabetic patients, first admitted for sepsis, were enrolled. Propensity score matching was conducted in a $1: 5$ ratio between pioglitazone users and nonusers. Multivariate logistic regression was conducted to evaluate the adjusted odds ratios (aORs) of hospital mortality in pioglitazone users. Further stratification analysis was done and Kaplan-Meier plot was used. Results. A total of 9,310 sepsis pioglitazone users (defined as "ever" use of pioglitazone in any dose within 3 months prior to the first admission for sepsis) and 46,550 matched nonusers were retrieved, respectively. In the multivariate logistic regression model, the cohort of pioglitazone users $(9,310)$ had a decreased aOR of 0.95 (95\% CI, 0.89-1.02) of sepsis mortality. Further stratification analysis demonstrated that "chronic pioglitazone users" (defined as "at least" 4-week drug use within 3 months) $(3,399)$ were more associated with significant aOR of 0.80 (95\% CI, 0.72-0.89) in reducing sepsis mortality. Conclusions. This first human cohort study demonstrated the potential protective effect of chronic pioglitazone use in type 2 diabetic sepsis patients.
\end{abstract}

\section{Introduction}

Sepsis is a major cause of mortality worldwide, especially in the immunocompromised patients, such as those with multiple comorbidities [1-3]. Sepsis is a complex syndrome that is induced by severe infection with a series of unregulated immune responses, caused majorly by the proinflammatory cytokines. Acute organ failure and subsequent high mortality rate will induce long-term morbidities, such as stroke and cardiovascular diseases [3,4]. Despite advances in treatment strategies, therapies to mitigate the severity of sepsis are currently unsatisfactory [5].

Thiazolidinediones (TZD), a kind of oral antidiabetic drugs (OADs), are used for the treatment of type 2 diabetes via being the insulin sensitizers [6]. Currently, pioglitazone is the only TZD available in the market. It is proposed to have a protection effect during the sepsis course by acting as the peroxisome proliferator-activated receptor-gamma 
(PPAR $\gamma$ ) agonist [7]. Because of multiple concerns about TZDs' complications, for example, cardiovascular disease and urinary bladder cancer, the prescriptions of TZD decreased significantly, from $9.20 \%$ in 2006 to $2.86 \%$ in 2012 in Taiwan [8-10].

Accumulating evidence in animal studies demonstrated that PPAR $\gamma$ agonists improved the outcomes of sepsis via multiple mechanisms [11]. In the mouse model, pioglitazone administration decreased inflammation and improved survival of sepsis induced by cecal ligation and puncture (CLP) [12]. Because of the growing amount of evidence, the randomized clinical trial of pioglitazone use in sepsis patients is underway [13]. However, currently, there are limited data on this topic in humans, especially in type 2 diabetic patients, because the varied levels of diabetic complication burdens in each person were not easily compared [14].

In the current study, we used a specially applied nationwide database of diabetic patients, from 1999 to 2013, with the first admission for sepsis to evaluate the impacts of pioglitazone use in sepsis with the main outcome of the total hospital mortality.

This cohort study addressed the selection bias from diabetic severity by using the propensity score matching and simulated a real-world clinical trial to compare subjects in each group.

\section{Methods}

2.1. Data Sources and Study Participants. We conducted this cohort study by using the National Health Insurance Research Database (NHIRD) of Taiwan. The National Health Insurance program in Taiwan currently provides coverage for more than $99 \%$ of the entire population. The National Health Insurance in Taiwan provides excellent healthcare service to the people [15-18]. The deidentified patient information and claims data were released to the National Health Research Institute to establish the NHIRD. The diagnosis codes of the International Classification of Diseases, Ninth Revision, Clinical Modification (ICD-9-CM) are used.

From the NHIRD, we conducted this study by using the specially applied database of "Longitudinal Cohort of Diabetes Patients (LHDB)" which enrolled a longitudinal cohort of 1.6 million newly diagnosed diabetic patients from 1999 to 2013. We retrieved data from LHDB to constitute the study and comparison cohorts, composed by type 2 diabetic patients with a first admission for sepsis with and without pioglitazone use.

2.2. Definition of Sepsis and Baseline Comorbidities. The diagnosis of sepsis in the current study was retrieved using the ICD-9-CM code 038 plus a main infection diagnosis with antibiotics prescription. The accuracy of sepsis diagnosis in the NHIRD has been validated [19]. The patients were defined as having certain comorbidities if they had at least 2 outpatient service claims or if they had a single hospitalization in which the certain comorbidities were found. The index date was defined as the first admission date for sepsis.
2.3. Definition of Drug Use in Pioglitazone. In this study, if a patient received the prescription of any dose of pioglitazone within 3 months prior to the index admission for sepsis, he or she would be defined as a pioglitazone user or "ever use" pioglitazone. Throughout the whole study, we used the above definition to describe any drug use.

To reduce the medical expenditure for the stable patients of type 2 diabetes or other chronic diseases, the physicians can use the refill card of consecutive prescription for 3 months rather than prescribing the drugs week by week or only 3 days. Based on the above medical regulation and culture in Taiwan, we therefore defined a person as a "chronic" pioglitazone user if he or she was prescribed pioglitazone for at least 4 weeks within 3 months prior to the first admission for sepsis [20].

2.4. Propensity Score Matching. Propensity score matching could reduce the selection bias because it allowed the bundling of many confounding factors which were frequently presented in the observation studies [21-23]. We calculated the propensity score using the multivariate logistic regression by entering the baseline covariates which included age, sex, comorbidities, insurance premium, and complication severity of type 2 DM.

Since type 2 diabetes related complications may be the most important factor to determine the hospital outcome, we matched 1 study cohort patient with 5 comparison cohort patients according to propensity score and obtained a dataset composed of matched patients who had a statistically identical likelihood of severity of diabetic complications.

In the database, the individual insurance premium fee paid was a useful surrogate for the household income level [24].

2.5. Selection Process. The algorithm used for participant selection for the study and comparison cohorts is shown in Figure 1 . Patients aged $<18$ or $>100$ years, patients with type 1 diabetes, and patients infected with human immunodeficiency virus were excluded from this study. Since the database contains deidentified data for research, our study was exempted from the requirement of informed consent from participants. This study was approved by the Institutional Review Board of Taipei Veterans General Hospital (2020-01-012CC) and China Medical University (CMUH104-REC2-115).

2.6. Comparison between the Study and Matched Cohorts. Differences in demographic characteristics, insurance premium, baseline comorbidities, medications (including nonsteroidal anti-inflammatory drugs (NSAIDs), aspirin, statins, biguanides, dipeptidyl peptidase-4 inhibitors (DPP-4 inhibitors), sulfonylureas, pioglitazone, insulin, immunosuppressants, and steroids), infection sites, adapted diabetes complications severity index (aDCSI) score which was a representation of diabetes complication severity, length of hospital stay, and the total hospital mortality were examined using the chi-squared test and two-sample $t$-test. 


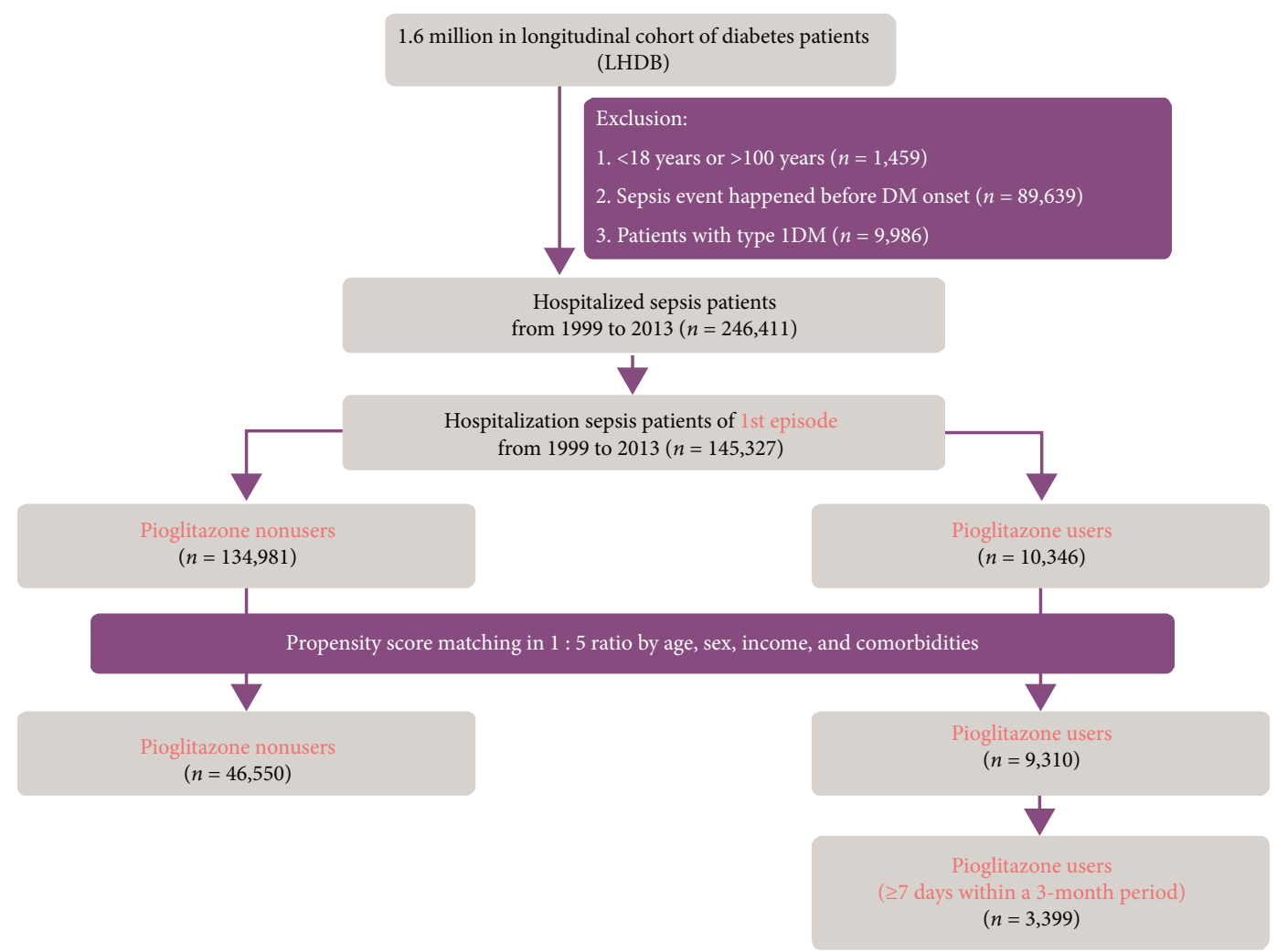

Figure 1: The participant selection process in the study and comparison cohorts.

2.7. Logistic Regression and Kaplan-Meier Analysis. Odds ratios (ORs) and 95\% confidence intervals (95\% CIs) were calculated for each variable in the logistic regression model. Adjusted ORs (aORs) for the total hospital mortality were obtained after adjusting for potential confounders, including age, sex, income, and comorbidities, in the multivariate logistic regression analysis. Kaplan-Meier analysis with logrank test was conducted to compare the difference in the outcomes of total hospital mortality between the study and comparison cohorts (i.e., pioglitazone users versus nonusers). The statistical analyses were performed using the SAS 9.4 statistical package (SAS Institute, Inc., Cary, NC, USA). A $P$ value of 0.05 was considered significant.

\section{Results}

3.1. Demographic Characteristics and Baseline Comorbidities between the Enrolled Sepsis Patients of Pioglitazone Users and Nonusers. From the LHDB, we initially retrieved a total of 145,327 type 2 diabetic patients with the first admission for sepsis from 1999 to 2013 . After propensity score matching, a total of 9,310 pioglitazone users and 46,550 nonusers were included for further analysis.

Before PS matching, the mean ages of pioglitazone users and nonusers were $67.08 \pm 12.62$ and $71.13 \pm 13.90$ years, respectively. After PS matching, the mean ages of pioglitazone users and nonusers were 68.11 \pm 12.50 and $68.96 \pm 13.18$ years, respectively. The detailed demographic characteristics are shown in Table 1. Before matching, a greater proportion of pioglitazone users than nonusers received treatment with statins (30.85\% versus $11.52 \%)$, biguanide $(60.48 \%$ versus $28.80 \%)$, DPP-4 inhibitors (21.35\% versus $3.52 \%)$, sulfonylurea (65.17\% versus $31.84 \%)$, and insulin $(68.11 \%$ versus $43.37 \%)$ (all $P<0.001)$. The pioglitazone users had fewer respiratory system infection sites compared to nonusers (46.24\% versus $51.76 \%)$ $(P<0.001)$. The pioglitazone users had more severe complication burdens (aDCSI score $\geq 5,16.30 \%$ of pioglitazone users versus $8.95 \%$ of nonusers $)(P<0.001)$. However, the pioglitazone users had lower total hospital mortality rate (15.83\% versus $18.6 \%$ ) and shorter length of hospital stay (mean, 11 versus 12 days) than the nonusers.

3.2. Regression Model of the Total Hospital Mortality. In the logistic regression model, after further adjusting for age, sex, income, and comorbidities, the pioglitazone users (ever use) were shown to have a nonsignificant aOR slightly less than unity for total hospital mortality $(\mathrm{aOR}=0.95$ (95\% CI, 0.89-1.03)) (Table 2).

In the further stratification analysis, the patients who were classified as "chronic pioglitazone users" demonstrated the significant aOR for total hospital mortality $(\mathrm{aOR}=0.80$ (95\% CI, 0.72-0.89), $P<0.05$ ) (Table 3).

3.3. Kaplan-Meier Analysis of the Total Hospital Mortality. In the Kaplan-Meier analysis with log-rank test, the total hospital mortality did not differ significantly between the pioglitazone users ("ever use") and nonusers (Figure 2). However, in the "chronic pioglitazone users," it was obvious 
TABle 1: Demographic characteristics, comorbidities, and diabetic complications of type 2 diabetic sepsis patients between pioglitazone users and nonusers.

\begin{tabular}{|c|c|c|c|c|c|c|c|c|c|c|c|c|}
\hline \multirow{3}{*}{ Variables } & \multicolumn{6}{|c|}{ Before matching } & \multicolumn{6}{|c|}{ After PS matching } \\
\hline & \multicolumn{2}{|c|}{$\begin{array}{c}\text { Nonusers } \\
(n=134,981)\end{array}$} & \multicolumn{2}{|c|}{$\begin{array}{c}\text { Users } \\
(n=10,346)\end{array}$} & \multirow{2}{*}{$\begin{array}{c}P^{*} \\
\text { value }\end{array}$} & \multirow{2}{*}{$\begin{array}{c}\text { Standardized } \\
\text { mean difference }\end{array}$} & \multicolumn{2}{|c|}{$\begin{array}{l}\text { Nonusers } \\
(n=46,550)\end{array}$} & \multicolumn{2}{|c|}{$\begin{array}{c}\text { Users } \\
(n=9,310)\end{array}$} & \multirow{2}{*}{$\begin{array}{c}P^{*} \\
\text { value }\end{array}$} & \multirow{2}{*}{$\begin{array}{l}\text { Standardized } \\
\text { mean difference }\end{array}$} \\
\hline & $n$ & $\%$ & $n$ & $\%$ & & & $n$ & $\%$ & $n$ & $\%$ & & \\
\hline \multirow[t]{3}{*}{ Sex } & & & & & $<0.001$ & & & & & & 0.361 & 0.010 \\
\hline & 61,623 & 45.65 & 5,018 & 48.50 & & 0.057 & 22,289 & 47.88 & 4,506 & 48.40 & & \\
\hline & 73,358 & 54.35 & 5,328 & 51.50 & & 0.057 & 24,261 & 52.12 & 4,804 & 51.60 & & \\
\hline Age, years & & & & & $<0.001$ & & & & & & 0.004 & \\
\hline $18-29$ & 600 & 0.44 & 12 & 0.12 & & 0.062 & 116 & 0.25 & 9 & 0.10 & & 0.037 \\
\hline $30-49$ & 11,323 & 8.39 & 994 & 9.61 & & 0.043 & 4,079 & 8.76 & 766 & 8.23 & & 0.019 \\
\hline $50-69$ & 42,086 & 31.18 & 4,718 & 45.60 & & 0.300 & 18,941 & 40.69 & 3,969 & 42.63 & & 0.039 \\
\hline $70-89$ & 74,625 & 55.29 & 4,468 & 43.19 & & 0.244 & 22,587 & 48.52 & 4,412 & 47.39 & & 0.023 \\
\hline $90-100$ & 6,347 & 4.70 & 154 & 1.49 & & 0.186 & 827 & 1.78 & 154 & 1.65 & & 0.009 \\
\hline Mean (SD) & \multicolumn{2}{|c|}{$71.13(13.90)$} & $\begin{array}{r}67 \\
(12 .\end{array}$ & $\begin{array}{l}.08 \\
.62) \\
\end{array}$ & $<0.001$ & 0.305 & 68.96 & 13.18) & $\begin{array}{r}68 . \\
(12 .\end{array}$ & $\begin{array}{l}.11 \\
.50) \\
\end{array}$ & $<0.001$ & 0.066 \\
\hline Insurance premium $\left(\mathrm{N}^{\prime}\right.$ & T dollars) & & & & & & & & & & 0.395 & \\
\hline$<20000$ & 82,771 & 61.32 & 4,705 & 45.48 & & 0.322 & 22,918 & 49.23 & 4,523 & 48.58 & & 0.013 \\
\hline $20000-40000$ & 43,501 & 32.23 & 4,575 & 44.22 & & 0.249 & 19,836 & 42.61 & 4,023 & 43.21 & & 0.012 \\
\hline $40000-60000$ & 6,461 & 4.79 & 800 & 7.73 & & 0.122 & 2,890 & 6.21 & 598 & 6.42 & & 0.009 \\
\hline$>60000$ & 2,248 & 1.67 & 266 & 2.57 & & 0.063 & 906 & 1.95 & 166 & 1.78 & & 0.012 \\
\hline Comorbidity & & & & & & & & & & & & \\
\hline HTN & 101,906 & 75.50 & 8,732 & 84.4 & $<0.001$ & 0.224 & 39,091 & 83.98 & 7,728 & 83.01 & 0.020 & 0.026 \\
\hline Hyperlipidemia & 62,096 & 46.00 & 7,397 & 71.5 & $<0.001$ & 0.536 & 31,910 & 68.55 & 6,363 & 68.35 & 0.698 & 0.004 \\
\hline COPD & 63,740 & 47.22 & 4,049 & 39.14 & $<0.001$ & 0.164 & 19,357 & 41.58 & 3,868 & 41.55 & 0.948 & 0.001 \\
\hline CLD & 45,715 & 33.87 & 4,091 & 39.54 & $<0.001$ & 0.118 & 18,691 & 40.15 & 3,674 & 39.46 & 0.215 & 0.014 \\
\hline CKD & 61,015 & 45.20 & 5,988 & 57.88 & $<0.001$ & 0.256 & 25,183 & 54.10 & 5,093 & 54.70 & 0.284 & 0.012 \\
\hline PAOD & 20,462 & 15.16 & 1,832 & 17.71 & $<0.001$ & 0.069 & 7,625 & 16.38 & 1,597 & 17.15 & 0.066 & 0.021 \\
\hline IHD & 64,874 & 48.06 & 5,144 & 49.72 & 0.011 & 0.033 & 23,337 & 50.13 & 4,668 & 50.14 & 0.990 & 0 \\
\hline Stroke & 60,780 & 45.03 & 4,343 & 41.98 & $<0.001$ & 0.062 & 19,974 & 42.91 & 4,063 & 43.64 & 0.192 & 0.015 \\
\hline Cancer & 38,145 & 28.26 & 2,693 & 26.03 & $<0.001$ & 0.050 & 12,649 & 27.17 & 2,541 & 27.29 & 0.811 & 0.003 \\
\hline Drug use & & & & & & & & & & & & \\
\hline NSAID & 74,050 & 54.86 & 5,534 & 53.49 & 0.070 & 0.028 & 26,508 & 56.95 & 4,968 & 53.36 & $<0.001$ & 0.072 \\
\hline Aspirin & 15,122 & 11.20 & 844 & 8.16 & $<0.001$ & 0.103 & 5,201 & 11.17 & 759 & 8.15 & $<0.001$ & 0.102 \\
\hline Statins & 15,549 & 11.52 & 3,192 & 30.85 & $<0.001$ & 0.487 & 7,585 & 16.29 & 2,763 & 29.68 & $<0.001$ & 0.322 \\
\hline Biguanides & 38,874 & 28.80 & 6,257 & 60.48 & $<0.001$ & 0.672 & 14,986 & 32.19 & 5,667 & 60.87 & $<0.001$ & 0.600 \\
\hline DPP-4 inhibitors & 4,751 & 3.52 & 2,209 & 21.35 & $<0.001$ & 0.561 & 2,145 & 4.61 & 1,932 & 20.75 & $<0.001$ & 0.500 \\
\hline Sulfonylureas & 42,982 & 31.84 & 6,743 & 65.17 & $<0.001$ & 0.707 & 16,255 & 34.92 & 6,098 & 65.50 & $<0.001$ & 0.642 \\
\hline Pioglitazone & 0 & 0 & 3,764 & 36.38 & - & - & 0 & 0 & 3,399 & 36.51 & $<0.001$ & 1.072 \\
\hline Insulin & 58,539 & 43.37 & 7,047 & 68.11 & $<0.001$ & 0.514 & 20,724 & 44.52 & 6,296 & 67.63 & $<.0001$ & 0.479 \\
\hline $\begin{array}{l}\text { Immuno } \\
\text { suppressants }\end{array}$ & 475 & 0.35 & 57 & 0.55 & 0.001 & 0.030 & 224 & 0.48 & 41 & 0.44 & 0.600 & 0.006 \\
\hline Steroids & 38,618 & 28.61 & 2,876 & 27.8 & 0.078 & 0.018 & 13,213 & 28.38 & 2,608 & 28.01 & 0.467 & 0.008 \\
\hline Infection site & & & & & & & & & & & & \\
\hline Central nervous & 1,411 & 1.05 & 100 & 0.97 & 0.446 & 0.008 & 525 & 1.13 & 88 & 0.95 & 0.122 & 0.018 \\
\hline Respiratory & 69,870 & 51.76 & 4,784 & 46.24 & $<0.001$ & 0.111 & 21,986 & 47.23 & 4,416 & 47.43 & 0.721 & 0.004 \\
\hline Cardiovascular & 1,938 & 1.44 & 156 & 1.51 & 0.553 & 0.006 & 656 & 1.41 & 133 & 1.43 & 0.885 & 0.002 \\
\hline Gastrointestinal & 20,361 & 15.08 & 1,795 & 17.35 & $<0.001$ & 0.061 & 7,459 & 16.02 & 1,596 & 17.14 & 0.007 & 0.030 \\
\hline Genitourinary & 69,360 & 51.39 & 5,472 & 52.89 & 0.003 & 0.030 & 23,449 & 50.37 & 4,959 & 53.27 & $<.001$ & 0.058 \\
\hline Soft tissue/bone & 24,980 & 18.51 & 2,262 & 21.86 & $<0.001$ & 0.084 & 8,782 & 18.87 & 1,991 & 21.39 & $<.001$ & 0.063 \\
\hline Device-related & 4,847 & 3.59 & 431 & 4.17 & 0.002 & 0.030 & 1,946 & 4.18 & 354 & 3.80 & 0.093 & 0.019 \\
\hline Others & 34,048 & 25.22 & 2,568 & 24.82 & 0.362 & 0.009 & 10,969 & 23.56 & 2,309 & 24.8 & 0.105 & 0.029 \\
\hline aDCSI score & & & & & $<0.001$ & & & & & & $<0.001$ & \\
\hline 0 & 38,882 & 28.81 & 2,092 & 20.22 & & 0.201 & 12,732 & 27.35 & 1,937 & 20.81 & & 0.154 \\
\hline 1 & 15,563 & 11.53 & 1,485 & 14.35 & & 0.084 & 5,818 & 12.50 & 1,319 & 14.17 & & 0.049 \\
\hline 2 & 36,729 & 27.21 & 2,288 & 22.11 & & 0.118 & 12,078 & 25.95 & 2,067 & 22.20 & & 0.088 \\
\hline 3 & 13,194 & 9.77 & 1,379 & 13.33 & & 0.111 & 4,844 & 10.41 & 1,243 & 13.35 & & 0.091 \\
\hline 4 & 18,532 & 13.73 & 1,416 & 13.69 & & 0.001 & 6,425 & 13.80 & 1,281 & 13.76 & & 0.001 \\
\hline$\geq 5$ & 12,081 & 8.95 & 1,686 & 16.30 & & 0.223 & 4,653 & 10.00 & 1,463 & 15.71 & & 0.171 \\
\hline $\begin{array}{l}\text { Length of hospital stay } \\
\text { (days) (median) }\end{array}$ & 12 & & 1 & 1 & & 0.056 & 11 & & 1 & 1 & 0.0215 & 0.027 \\
\hline $\begin{array}{l}\text { Total hospital } \\
\text { mortality }\end{array}$ & 25,102 & 18.6 & 1,638 & 15.83 & $<0.001$ & 0.073 & 7,947 & 17.07 & 1,523 & 16.36 & 0.0941 & 0.019 \\
\hline
\end{tabular}


TABLE 2: Logistic regression model to estimate the OR and 95\% CI of the total hospital mortality in pioglitazone users and nonusers.

\begin{tabular}{|c|c|c|}
\hline \multirow[b]{2}{*}{ Variable } & \multicolumn{2}{|c|}{ Outcome $=$ total hospital mortality } \\
\hline & $\begin{array}{c}\text { Crude OR } \\
(95 \% \mathrm{CI})\end{array}$ & $\begin{array}{c}\text { Adjusted OR } \\
(95 \% \mathrm{CI})\end{array}$ \\
\hline Pioglitazone use & $0.95(0.88-1.01)$ & $0.95(0.89-1.03)$ \\
\hline \multicolumn{3}{|l|}{$\operatorname{Sex}$} \\
\hline Female & 1 (ref) & 1 (ref) \\
\hline Male & $1.59(1.52-1.66)^{*}$ & $1.54(1.47-1.62)^{*}$ \\
\hline \multicolumn{3}{|l|}{ Age, years } \\
\hline $18-29$ years & 1 (ref) & 1 (ref) \\
\hline $30-49$ years & $1.45(0.78-2.70)$ & $1.39(0.74-2.63)$ \\
\hline $50-69$ years & $1.94(1.04-3.60)^{*}$ & $1.67(0.89-3.13)^{*}$ \\
\hline $70-89$ years & $2.36(1.27-4.39)^{*}$ & $1.93(1.03-3.62)^{*}$ \\
\hline $90-100$ years & $3.34(1.77-6.30)^{*}$ & $2.85(1.49-5.45)^{*}$ \\
\hline \multicolumn{3}{|c|}{ Insurance premium (NT dollars) } \\
\hline$<20000$ & 1 (ref) & 1 (ref) \\
\hline $20000-40000$ & $0.60(0.58-0.63)^{*}$ & $0.61(0.58-0.64)^{*}$ \\
\hline $40000-60000$ & $0.66(0.60-0.73)^{*}$ & $0.64(0.58-0.71)^{*}$ \\
\hline$>60000$ & $0.92(0.79-1.08)$ & $0.76(0.65-0.90)^{*}$ \\
\hline \multicolumn{3}{|c|}{ Baseline comorbidity $($ Ref $=$ Non- $)$} \\
\hline HTN & $1.00(0.94-1.06)$ & $0.95(0.88-1.01)$ \\
\hline Hyperlipidemia & $0.77(0.73-0.80)^{*}$ & $0.77(0.74-0.81)^{*}$ \\
\hline COPD & $0.96(0.92-1.01)$ & $0.84(0.80-0.88)^{*}$ \\
\hline CLD & $1.14(1.09-1.19)^{*}$ & $1.06(1.01-1.11)^{*}$ \\
\hline CKD & $1.38(1.32-1.45)^{*}$ & $1.35(1.29-1.42)^{*}$ \\
\hline PAOD & $1.16(1.09-1.22)^{*}$ & $1.11(1.05-1.18)^{*}$ \\
\hline IHD & $1.02(0.97-1.06)$ & $0.99(0.95-1.04)$ \\
\hline Stroke & $0.99(0.94-1.03)$ & $0.96(0.91-1.00)$ \\
\hline Cancer & $3.29(3.14-3.44)^{*}$ & $3.23(3.08-3.39)^{*}$ \\
\hline
\end{tabular}

Adjusted OR: adjusted for age, sex, insurance premium, and comorbidities in logistic regression. Any dose of pioglitazone within 3 months prior to the index admission for sepsis; ${ }^{*} P<0.05$. CI, confidence interval; CKD, chronic kidney disease; CLD, chronic liver disease; COPD, chronic obstructive pulmonary disease; DPP-4 inhibitor, dipeptidyl peptidase- 4 inhibitor; HTN, hypertension; IHD, ischemic heart disease; NSAID, nonsteroidal anti-inflammatory drug; NT, new Taiwan; OR, odds ratio; PAOD, peripheral arterial occlusion disease.

that the cumulative survival rate was much better than that in the nonusers $(P<0.01)$ (Figure 3$)$.

\section{Discussion}

In this real-world study, by using the nationwide database of diabetic patients with propensity score matching, we demonstrated that pioglitazone use can exert a significantly protective effect in "chronic pioglitazone users." This finding has been proved in multiple animal studies for a long term. However, it remained controversial in human beings. To the best of our knowledge, this is the first and largest cohort study of type 2 diabetic sepsis patients that simulated the human clinical trial via propensity score matching to examine the protective effect of pioglitazone. Our finding will surely attract more and more attention focusing on the potential of pioglitazone in sepsis.

PPARs encoded by separate genes, PPAR $\alpha, \operatorname{PPAR} \beta / \delta$, and PPAR $\gamma$, are expressed by a variety of cells of the immune system including macrophages, $B$ and $T$, and monocytes, lymphocyte, natural killer cells, dendritic cells,
TABLE 3: Logistic regression model to estimate the OR and 95\% CI of total hospital mortality in pioglitazone users ( $\geq 7$ days within 3 months) and nonusers.

\begin{tabular}{|c|c|c|}
\hline \multirow[b]{2}{*}{ Variable } & \multicolumn{2}{|c|}{ Outcome $=$ total hospital mortality } \\
\hline & $\begin{array}{c}\text { Crude OR } \\
(95 \% \text { CI })\end{array}$ & $\begin{array}{c}\text { Adjusted OR } \\
(95 \% \mathrm{CI})\end{array}$ \\
\hline Pioglitazone & $0.76(0.69-0.84)^{*}$ & $0.80(0.72-0.89)^{*}$ \\
\hline \multicolumn{3}{|l|}{ Sex } \\
\hline Female & 1 (reference) & 1 (reference) \\
\hline Male & $1.59(1.52-1.66)^{*}$ & $1.54(1.47-1.62)$ \\
\hline \multicolumn{3}{|l|}{ Age, years } \\
\hline $18-29$ years & 1 (ref) & 1 (ref) \\
\hline $30-49$ years & $1.45(0.78-2.70)$ & $1.40(0.74-2.64)$ \\
\hline $50-69$ years & $1.94(1.04-3.60)^{*}$ & $1.67(0.89-3.14)$ \\
\hline $70-89$ years & $2.36(1.27-4.39)^{*}$ & $1.94(1.03-3.64)$ \\
\hline $90-100$ years & $3.34(1.77-6.30)^{*}$ & $2.85(1.49-5.46)$ \\
\hline \multicolumn{3}{|c|}{ Insurance premium (NT dollars) } \\
\hline$<20000$ & 1 (ref) & 1 (ref) \\
\hline $20000-40000$ & $0.60(0.58-0.63)^{*}$ & $0.61(0.58-0.64)$ \\
\hline $40000-60000$ & $0.66(0.60-0.73)^{*}$ & $0.64(0.58-0.71)$ \\
\hline$>60000$ & $0.92(0.79-1.08)$ & $0.76(0.65-0.90)$ \\
\hline \multicolumn{3}{|c|}{ Comorbidity (Ref = Non-) } \\
\hline HTN & $1.00(0.94-1.06)$ & $0.95(0.88-1.01)$ \\
\hline Hyperlipidemia & $0.77(0.73-0.80)^{*}$ & $0.77(0.74-0.81)$ \\
\hline COPD & $0.96(0.92-1.01)$ & $0.84(0.80-0.88)$ \\
\hline CLD & $1.14(1.09-1.19)^{*}$ & $1.06(1.01-1.11)$ \\
\hline CKD & $1.38(1.32-1.45)^{*}$ & $1.35(1.29-1.42)$ \\
\hline PAOD & $1.16(1.09-1.22)^{*}$ & $1.11(1.05-1.18)$ \\
\hline IHD & $1.02(0.97-1.06)$ & $0.99(0.94-1.04)$ \\
\hline Stroke & $0.99(0.94-1.03)$ & $0.96(0.91-1.00)$ \\
\hline Cancer & $3.29(3.14-3.44)^{*}$ & $3.23(3.08-3.38)$ \\
\hline
\end{tabular}

Adjusted OR: adjusted for age, sex, insurance premium, and comorbidities in logistic regression. ${ }^{*} P<0.05 ; \geq 7$ days within 3 months prior to the index admission for sepsis. CI, confidence interval; $\mathrm{CKD}$, chronic kidney disease; CLD, chronic liver disease; COPD, chronic obstructive pulmonary disease; DPP-4 inhibitor, dipeptidyl peptidase-4 inhibitor; HTN, hypertension; IHD, ischemic heart disease; NSAID, nonsteroidal anti-inflammatory drug; NT, new Taiwan; OR, odds ratio; PAOD, peripheral arterial occlusion disease.

mass cells, neutrophils, and eosinophils [25]. PPARs have received attention till now, since they play pivotal regulators in adipocyte differentiation, glucose homeostasis, and immune regulation. In the current study, we focus on the role of immune modification of pioglitazone which is a kind of TZDs, activating as the PPAR $\gamma$ agonist.

$\operatorname{PPAR} \gamma$ agonists can be simply classified into natural and artificial ones, respectively. Natural PPAR $\gamma$ agonists include saturated and unsaturated fatty acids, eicosanoid derivatives, such as 15-deoxy- $\Delta^{12,14}$-prostaglandin J2 (15d-PGJ2), and oleic and nitrated linoleic acids. Synthetic PPAR $\gamma$ agonists are represented by TZDs, such as pioglitazone, rosiglitazone, troglitazone, and ciglitazone. TZDs (agonist) function via activating the PPAR $\gamma$ receptor $[26,27]$. In the absence of $\operatorname{PPAR} \gamma$ agonists, these ligands remain inactive via binding to the corepressors.

Pioglitazone is currently the only available TZD in the market, since rosiglitazone has been suspended in Taiwan in 2011 due to its potentially increased risk of myocardial infarction and decompensated heart failure [28]. Pioglitazone targets the transcription of PPAR $\gamma$ and is involved in 


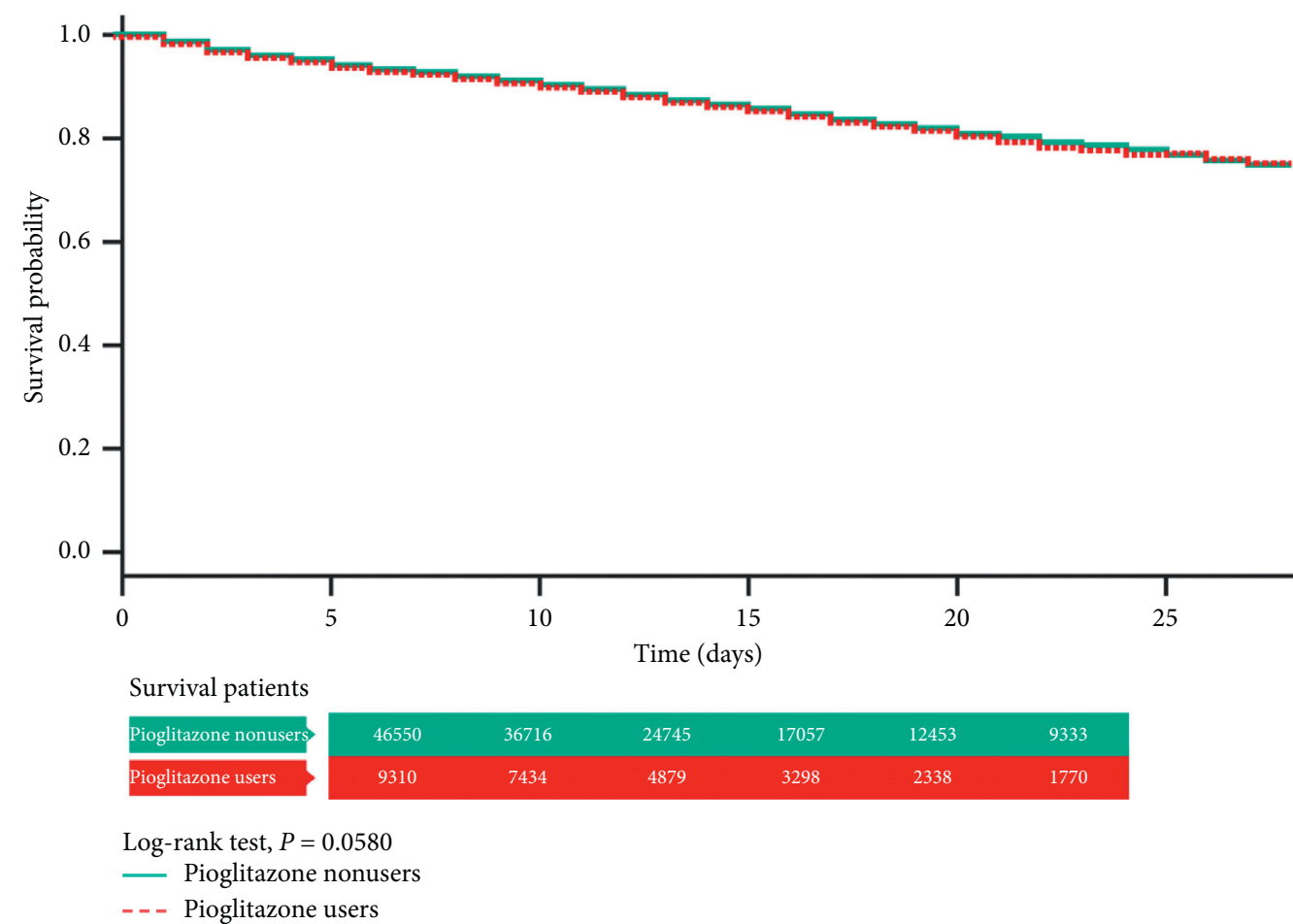

FIGURE 2: Kaplan-Meier analysis with log-rank test of the total hospital mortality in pioglitazone users (in any dose within 3 months prior to the first admission for sepsis) and nonusers.

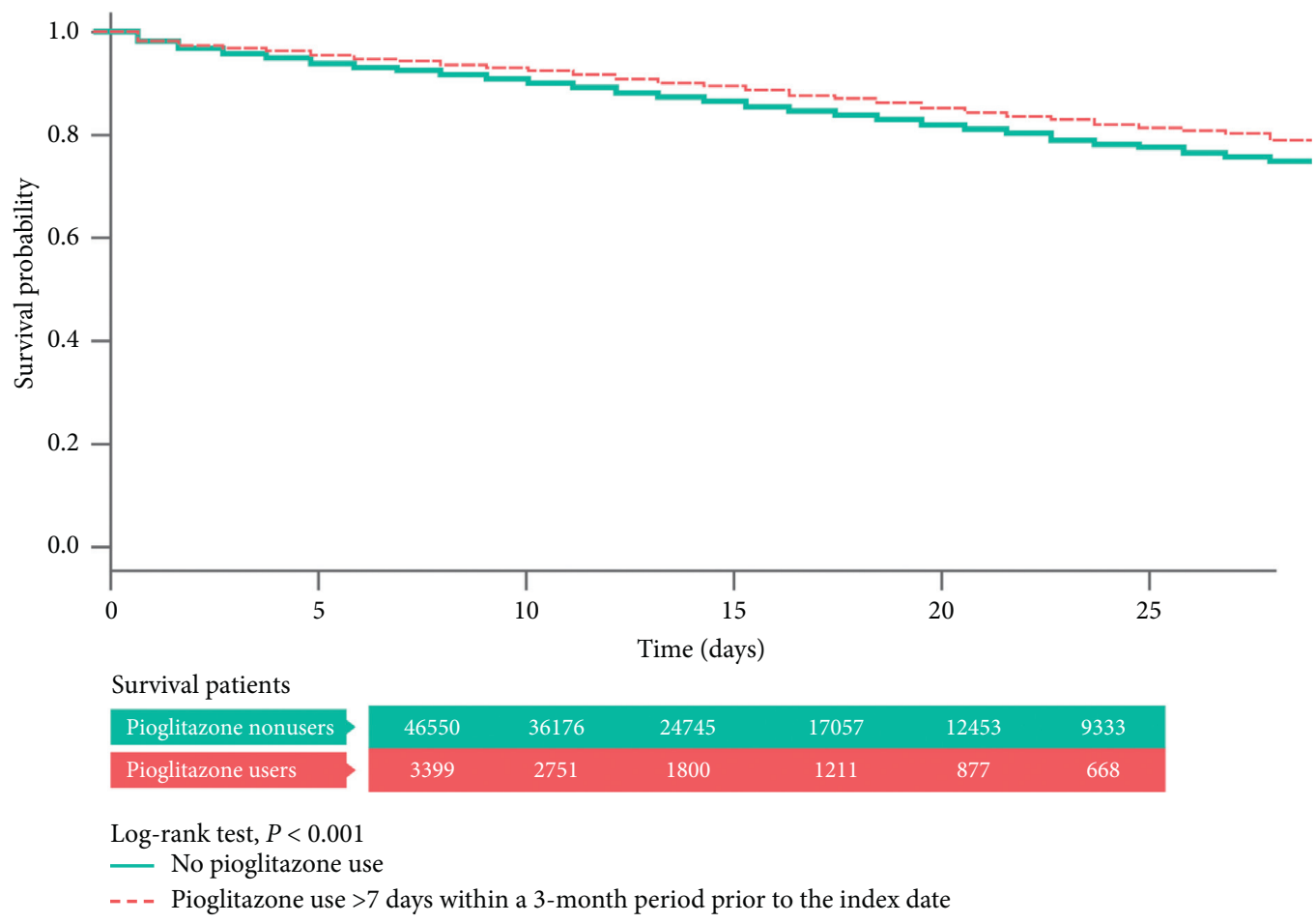

Figure 3: Kaplan-Meier analysis with log-rank test of the total hospital mortality in pioglitazone users (at least 4 weeks' drug use within 3 months) and nonusers.

metabolic homeostasis, and, most important of all, it much improves insulin sensitivity. This mechanism provides a choice to type 2 diabetic patients before receiving insulin injection therapy. Following the suspension of rosiglitazone in 2011, pioglitazone later became another target of criticism, including that pioglitazone (1) increased the risk of 
osteoporosis and (2) increased the risk of urinary bladder cancer. However, supporting data to pioglitazone passed these [29, 30]. Moreover, many studies and specialist opinion support that pioglitazone should continue to be used in T2D treatment [31-33].

In addition to helping glucose control, the activation of PPAR $\gamma$ agonist of pioglitazone contributes to the modulation of inflammation [34]. Pioglitazone improving bacterial elimination in the peripheral blood, via inhibition of proinflammatory molecules such as IL-6, TNF, IL-1, and IL-12, has been well documented. It also enhanced bacterial elimination in the liver by increasing the phagocytic and bactericidal activities [11]. Furthermore, accumulated evidence of animal models demonstrated that pioglitazone is effective in the prevention and treatment of sepsis in mice cecal ligation and puncture (CLP) model [35-37]. In the type 2 diabetic patients, who are prone to infection diseases, TZDs use with pioglitazone should be an adequate choice via the anti-inflammatory and enhancing bactericidal effects. Our study demonstrated the protective effect in sepsis patients, which added a new important evidence in human body.

The repeated or accumulated doses of pioglitazone presented the dose-effect relationship compared with the single-dose use as observed in this study. Besides, PPAR $\gamma$ agonists are known to upregulate their receptors' expression, which render the greater anticipated effects of repeated or accumulating dosing [38]. However, currently, which is the optimal dose and which stage to start pioglitazone treatment in sepsis deserve further investigation. Combining with other studies, we inferred that the initial or chronic use of pioglitazone might inhibit the cytokine storm and therefore reduced the acute organ failure in the first fulminant stage. Continuous use during the sepsis course remains for further study.

4.1. Limitations. This study has several limitations. First, this study lacked certain important laboratory data, that is, initial blood glucose level and hemoglobin A1C (HbA1c), which was an inevitable weak point in administrative database studies. However, we had demonstrated that there was no association between initial blood glucose, HbAlc, and hospital outcomes of sepsis in our previous hospital-based study; the lack of initial blood glucose level and HbA1c may be not as important as previously thought [14]. Second, the impact of pioglitazone use on every sepsis stage remains further examined since systemic inflammatory response syndrome (SIRS) and subsequent compensatory anti-inflammatory response syndrome (CARS) might occur in sequence or concurrently, named as mixed antagonist response syndrome (MARS). Our study design mainly focused on the first stage, that is, SIRS.

\section{Conclusion}

In this study, we demonstrated that, currently, regular preadmission pioglitazone use improved the total hospital mortality in type 2 diabetic sepsis patients after considering multiple variables, including comorbidities and household income.

\section{Data Availability}

The data that support the findings of this study were taken from the LHDB, but restrictions apply to the availability of these data, which were used under license for the current study. So the data are not publicly available. The data are however available from the authors upon reasonable request.

\section{Additional Points}

Highlights. (1) This nationwide database in Taiwan which included 1.6 million diabetic patients from 1999 to 2013 was used to examine the potential protective effect of pioglitazone in type 2 diabetic sepsis patients. (2) Propensity score matching to avoid selection bias and aDCSI score (representation of DM severity) to evaluate diabetes severity were addressed to retrieve the pioglitazone users $(9,310)$ and nonusers $(46,550)$ as the study and comparison cohort. (3) To the best of our knowledge, this is the first and largest cohort study to examine the potentially protective effects from pioglitazone use in sepsis, which was limited to animal studies. (4) In the stratification analysis, the study results demonstrated that pioglitazone use (at least 7 days within 3 months) was significantly associated with decreased mortality in sepsis patients.

\section{Ethical Approval}

This study was conducted by using the Longitudinal Cohort of Diabetes Patients (LHDB) in Taiwan. The LHDB contains deidentified secondary data for research. This study was approved by the Institutional Review Board of Taipei Veterans General Hospital (2020-01-012CC) and China Medical University (CMUH104-REC2-115).

\section{Conflicts of Interest}

The authors declare that there are no conflicts of interest.

\section{Authors' Contributions}

Ming-Shun Hsie, Sung-Yuan $\mathrm{Hu}$, and Chia-Ming Chang contributed to the conception and design. Vivian Chia-Rong Hsieh and Shu-Hui Liao performed data analysis and interpretation. Ming-Shun Hsieh wrote the manuscript. Chorng-Kuang How gave the final approval and performed critical revision. All the authors read and approved the final manuscript. Vivian Chia-Rong Hsieh and Chorng-Kuang How contributed equally to this study.

\section{Acknowledgments}

The authors acknowledge the Biostatistics Task Force of Taichung Veterans General Hospital, Taichung, Taiwan, for their assistance and advice in the statistical analyses. They also acknowledge the Clinical Informatics Research and 
Development Center of Taichung Veterans General Hospital, Taichung, Taiwan, for their assistance in data retrieval from the electronic database and further classification.

\section{Supplementary Materials}

Supplementary Table 1: infection site classification by ICD9-CM and ICD-10-CM. (Supplementary Materials)

\section{References}

[1] J.-L. Vincent, J. C. Marshall, S. A. Ñamendys-Silva et al., "Assessment of the worldwide burden of critical illness: the intensive care over nations (ICON) audit," The Lancet Respiratory Medicine, vol. 2, no. 5, pp. 380-386, 2014.

[2] C. Fleischmann, A. Scherag, N. K. J. Adhikari et al., "Assessment of global incidence and mortality of hospital-treated sepsis. current estimates and limitations," American Journal of Respiratory and Critical Care Medicine, vol. 193, no. 3, pp. 259-272, 2016.

[3] M. Singer, C. S. Deutschman, C. W. Seymour et al., "The third international consensus definitions for sepsis and septic shock (sepsis-3)," JAMA, vol. 315, no. 8, pp. 801-810, 2016.

[4] S.-M. Ou, H. Chu, P.-W. Chao et al., "Long-term mortality and major adverse cardiovascular events in sepsis survivors. a nationwide population-based study," American Journal of Respiratory and Critical Care Medicine, vol. 194, no. 2, pp. 209-217, 2016.

[5] R. P. Wenzel and M. B. Edmond, "Septic shock-evaluating another failed treatment," New England Journal of Medicine, vol. 366, no. 22, pp. 2122-2124, 2012.

[6] N. Kubota, Y. Terauchi, T. Kubota et al., "Pioglitazone ameliorates insulin resistance and diabetes by both adiponectin-dependent and -independent pathways," Journal of Biological Chemistry, vol. 281, no. 13, pp. 8748-8755, 2006.

[7] M. V. Carvalho, C. F. Gonçalves-de-Albuquerque, and A. R. Silva, "PPAR gamma: from definition to molecular targets and therapy of lung diseases," International Journal of Molecular Sciences, vol. 22, no. 2, 2021.

[8] S. E. Nissen and K. Wolski, "Effect of rosiglitazone on the risk of myocardial infarction and death from cardiovascular causes," New England Journal of Medicine, vol. 356, no. 24, pp. 2457-2471, 2007.

[9] L. Azoulay, H. Yin, K. B. Filion et al., "The use of pioglitazone and the risk of bladder cancer in people with type 2 diabetes: nested case-control study," BMJ, vol. 344, Article ID e3645, 2012.

[10] W.-M. Chu, H.-E. Ho, K.-H. Huang et al., "The prescribing trend of oral antidiabetic agents for type 2 diabetes in Taiwan," Medicine, vol. 96, no. 43, Article ID e8257, 2017.

[11] M. Nakashima, M. Kinoshita, H. Nakashima et al., "Pioglitazone improves phagocytic activity of liver recruited macrophages in elderly mice possibly by promoting glucose catabolism," Innate Immunity, vol. 25, no. 6, pp. 356-368, 2019.

[12] G. Haraguchi, H. Kosuge, Y. Maejima et al., "Pioglitazone reduces systematic inflammation and improves mortality in apolipoprotein E knockout mice with sepsis," Intensive Care Medicine, vol. 34, no. 7, pp. 1304-1312, 2008.

[13] J. M. Kaplan, B. Zingarelli, K. Krallman et al., "Phase 1 safety and pharmacokinetic study on the use of pioglitazone in critically ill patients with sepsis: a randomized clinical trial," Intensive Care Medicine, vol. 44, no. 11, pp. 2006-2008, 2018.
[14] M. S. Hsieh, S. Y. Hu, C. K. How et al., "Hospital outcomes and cumulative burden from complications in type 2 diabetic sepsis patients: a cohort study using administrative and hospital-based databases," Therapeutic Advances in Endocrinology and Metabolism, vol. 10, Article ID 2042018819875406, 2019.

[15] S.-J. Weng, D. Gotcher, H.-H. Wu, Y.-Y. Xu, C.-W. Yang, and L.-S. Lai, "Cloud image data center for healthcare network in Taiwan," Journal of Medical Systems, vol. 40, no. 4, p. 89, 2016.

[16] J. Schwartz, C. C. King, and M. Y. Yen, "Protecting health care workers during the COVID-19 coronavirus outbreak -lessons from Taiwan's SARS response," Clinical Infectious Diseases: An Official Publication of the Infectious Diseases Society of America, vol. 71, no. 15, 2020.

[17] H.-Y. Cheng, S.-Y. Li, and C.-H. Yang, "Initial rapid and proactive response for the COVID-19 outbreak - Taiwan's experience," Journal of the Formosan Medical Association, vol. 119, no. 4, pp. 771-773, 2020.

[18] C. Lin, W. E. Braund, J. Auerbach et al., "Policy decisions and use of information technology to fight 2019 novel coronavirus disease, Taiwan," Emerging Infectious Diseases, vol. 26, no. 7, 2020.

[19] P.-W. Chao, C.-J. Shih, Y.-J. Lee et al., "Association of postdischarge rehabilitation with mortality in intensive care unit survivors of sepsis," American Journal of Respiratory and Critical Care Medicine, vol. 190, no. 9, pp. 1003-1011, 2014.

[20] C.-Y. Wu, Y.-J. Chen, H. J. Ho et al., "Association between nucleoside analogues and risk of hepatitis $\mathrm{B}$ virus-related hepatocellular carcinoma recurrence following liver resection," JAMA, vol. 308, no. 18, pp. 1906-1914, 2012.

[21] P. C. Austin, "An introduction to propensity score methods for reducing the effects of confounding in observational studies," Multivariate Behavioral Research, vol. 46, no. 3, pp. 399-424, 2011.

[22] R. Qin, M. G. Titler, L. L. Shever, and T. Kim, "Estimating effects of nursing intervention via propensity score analysis," Nursing Research, vol. 57, no. 6, pp. 444-452, 2008.

[23] P. C. Austin, "Assessing balance in measured baseline covariates when using many-to-one matching on the propensity-score," Pharmacoepidemiology and Drug Safety, vol. 17, no. 12, pp. 1218-1225, 2008.

[24] M. S. Hsieh, C. K. How, V. C. Hsieh, and P. C. Chen, "Preadmission antihypertensive drug use and sepsis outcome: impact of angiotensin-converting enzyme inhibitors (ACEIs) and angiotensin receptor blockers (ARBs)," Shock, vol. 53, no. 4, pp. 407-415, 2019.

[25] B. Kota, T. Huang, and B. Roufogalis, "An overview on biological mechanisms of PPARs," Pharmacological Research, vol. 51, no. 2, pp. 85-94, 2005.

[26] F. Picard and J. Auwerx, "PPAR $(\gamma)$ and glucose homeostasis," Annual Review of Nutrition, vol. 22, no. 1, pp. 167-197, 2002.

[27] Y. Li, J. Zhang, F. J. Schopfer et al., "Molecular recognition of nitrated fatty acids by PPAR $\gamma$," Nature Structural \& Molecular Biology, vol. 15, no. 8, pp. 865-867, 2008.

[28] S. E. Aspinall, X. Zhao, C. B. Good, R. A. Stone, K. J. Smith, and F. E. Cunningham, "FDA warning and removal of rosiglitazone from VA national formulary," The American Journal of Managed Care, vol. 19, no. 9, pp. 748-758, 2013.

[29] J. D. Lewis, A. Ferrara, T. Peng et al., "Risk of bladder cancer among diabetic patients treated with pioglitazone: interim report of a longitudinal cohort study," Diabetes Care, vol. 34, no. 4, pp. 916-922, 2011. 
[30] C.-H. Tseng, "Pioglitazone and bladder cancer: a populationbased study of Taiwanese," Diabetes Care, vol. 35, no. 2, pp. 278-280, 2012.

[31] R. A. DeFronzo, S. Inzucchi, M. Abdul-Ghani, and S. E. Nissen, "Pioglitazone: the forgotten, cost-effective cardioprotective drug for type 2 diabetes," Diabetes and Vascular Disease Research, vol. 16, no. 2, pp. 133-143, 2019.

[32] R. E. J. Ryder, "Pioglitazone has a dubious bladder cancer risk but an undoubted cardiovascular benefit," Diabetic Medicine, vol. 32, no. 3, pp. 305-313, 2015.

[33] P. Korhonen, E. M. Heintjes, R. Williams et al., "Pioglitazone use and risk of bladder cancer in patients with type 2 diabetes: retrospective cohort study using datasets from four European countries," BMJ, vol. 354, Article ID i3903, 2016.

[34] B. Zingarelli and J. A. Cook, "Peroxisome proliferator-activated receptor-gamma is a new therapeutic target in sepsis and inflammation," Shock, vol. 23, no. 5, pp. 393-399, 2005.

[35] J. Kaplan, M. Nowell, R. Chima, and B. Zingarelli, "Pioglitazone reduces inflammation through inhibition of NF- $\kappa \mathrm{B}$ in polymicrobial sepsis," Innate Immunity, vol. 20, no. 5, pp. 519-528, 2014.

[36] Y. Tsujimura, T. Matsutani, A. Matsuda et al., "Effects of pioglitazone on survival and omental adipocyte function in mice with sepsis induced by cecal ligation and puncture," Journal of Surgical Research, vol. 171, no. 2, pp. e215-e221, 2011.

[37] J. M. Kaplan and B. Zingarelli, "Novel therapeutic agents in pediatric sepsis: peroxisome proliferator receptor $\gamma(\operatorname{PPAR} \gamma)$ agonists," The Open Inflammation Journal, vol. 4, no. Suppl 1M14, pp. 120-124, 2011.

[38] S. P. Lakshmi, A. T. Reddy, Y. Zhang et al., "Down-regulated peroxisome proliferator-activated receptor $\gamma(\operatorname{PPAR} \gamma)$ in lung epithelial cells promotes a PPAR $\gamma$ agonist-reversible proinflammatory phenotype in chronic obstructive pulmonary disease (COPD)," Journal of Biological Chemistry, vol. 289, no. 10, pp. 6383-6393, 2014. 